\title{
A Pilot Study using Covert Visuospatial Attention as an EEG-based Brain Computer Interface to Enhance AR Interaction
}

\author{
Nataliya Kosmyna \\ MIT Media Lab, Cambridge, \\ Massachusetts, United States \\ nkosmyna@mit.edu \\ Qiuxuan $\mathrm{Wu}$ \\ University of Chicago, Chicago, \\ Illinois, United States \\ eileenwqx@gmail.com
}

\author{
Chi-Yun $\mathrm{Hu}$ \\ Tufts University, Medford, \\ Massachusetts, United States \\ ChiaYun.Hu@tufts.edu \\ Cassandra Scheirer \\ Carnegie Mellon University, \\ Pittsburgh, Pennsylvania, United \\ States \\ cscheire@andrew.cmu.edu
}

\author{
Yujie Wang \\ MIT, Cambridge, Massachusetts, \\ United States \\ yujiew@mit.edu \\ Pattie Maes \\ MIT Media Lab, Cambridge, \\ Massachusetts, United States \\ pattie@media.mit.edu
}

\begin{abstract}
In this work we propose a prototype which combines an existing augmented reality (AR) headset, the Microsoft HoloLens 2, with an electroencephalogram (EEG) Brain-Computer Interface (BCI) system based on covert visuospatial attention (CVSA) - a process of focusing attention on different regions of the visual field without overt eye movements. In this work we did not rely on any stimulusdriven responses. Fourteen participants were able to test the system over the course of two days. To the best of our knowledge, this system is the first AR EEG-BCI integrated prototype that explores the complementary features of the AR headset like HoloLens 2 and the CVSA paradigm.
\end{abstract}

\section{CCS CONCEPTS}

- Human-centered computing $\rightarrow$ Human computer interaction (HCI); Interaction paradigms; Mixed / augmented reality.

\section{KEYWORDS}

Brain-Computer Interfaces, EEG, Attention, AR, HoloLens, Headset, CVSA

\section{ACM Reference Format:}

Nataliya Kosmyna, Chi-Yun Hu, Yujie Wang, Qiuxuan Wu, Cassandra Scheirer, and Pattie Maes. 2021. A Pilot Study using Covert Visuospatial Attention as an EEG-based Brain Computer Interface to Enhance AR Interaction. In 2021 International Symposium on Wearable Computers (ISWC '21), September 21-26, 2021, Virtual, USA. ACM, New York, NY, USA, 5 pages. https://doi.org/10.1145/3460421.3480420

\section{INTRODUCTION}

Research in the field of physiological sensing, and Brain-Computer Interfaces in particular, has been gaining momentum within the past 15 years, as the systems have been tested in scenarios such as rehabilitation [1], robotics [2], accessing mental states of the

\section{(c) (i) (2)}

This work is licensed under a Creative Commons

Attribution-NonCommercial-ShareAlike International 4.0 License.

ISWC '21, September 21-26, 2021, Virtual, USA

(c) 2021 Copyright held by the owner/author(s).

ACM ISBN 978-1-4503-8462-9/21/09.

https://doi.org/10.1145/3460421.3480420 user [3] and entertainment [4]. However, these systems remain expensive, bulky, uncomfortable to wear due to the gel applied to the electrodes, wired, as well as prone to classification errors. Thus, a lot of modern BCI systems are used in association with other input modalities like gaze trackers [5], or HMDs like Virtual Reality (VR) [6] and Augmented Reality (AR) headsets [7]. In this paper we focus on AR HMDs.

The connection between BCIs and AR HMDs is threefold: both $\mathrm{BCI}$ and $\mathrm{AR}$ systems are worn on the head; HMDs usually require hands-free interaction, which BCIs can provide; and AR HMD devices have voice and/or eye gaze modalities for interaction, which could compensate for classification errors that occur during BCI use.

However, these research works generally do not integrate the $\mathrm{BCI}$ and $\mathrm{AR} \mathrm{HMD}$ systems within the same physical prototype; thus, they barely address the challenges of using BCIs and AR HMDs simultaneously.

In this work we propose a prototype to solve this problem by combining an AR headset with an existing EEG-BCI system, and we conducted a study with 14 users, who were wearing our prototype to validate this concept (Fig. 1). We investigated the feasibility of using a BCI based on covert visuospatial attention (CVSA) - a process of focusing attention on different regions of the visual field without overt eye movements [8]. In this work we operate without relying on any stimulus-driven responses. We invited the users to test the system over a period of 2 days, and we report on our results in this paper.

Our contributions in this paper are (1) an AR EEG-BCI integrated prototype (2) a user study which is built upon CVSA paradigm (3) sharing our code as well as all pertaining 3D files to print a clipon holder for EEG electrodes and electronics compatible with AR HoloLens 2 headset [9], which we use in this work.

\subsection{Covert Visuospatial Attention (CVSA)}

Covert visuospatial attention (CVSA) is defined as the ability to allocate one's attention resources to a position located in one's peripheral field of view without any overt eye movements [8]. Since covert shifts of attention do not generate any observable behavior, EEG provides access to this process that cannot be measured through any other modality. Covert attention has been shown to be underlain by specific neurophysiological patterns, specifically by a 

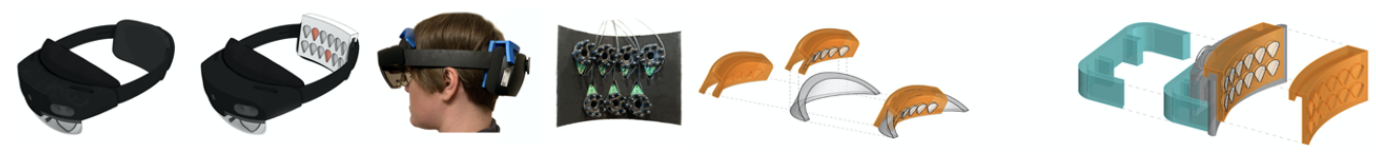

Figure 1: From left to right: Original HoloLens 2 AR headset (H2); HoloLens 2 headset with embedded brain sensing component (clip-on holder to house EEG electrodes over visual cortex, 2 EEG electrodes highlighted in orange); User wearing an "enhanced" AR/BCI system (clip-on holder printed in blue; over visual and pre-frontal cortex); A close-up image of the electrode clip-on featuring 7 electrodes; EEG clip-on hardware piece designed for HoloLens 2 headset. The clip-on piece includes three parts: the first two parts house EEG electrodes that make contact with the forehead (left image, highlighted in orange) and the back of the head (right image, highlighted in orange), while the last part, attached at the back of the HoloLens 2, holds the electronics (right image, highlighted in light green).

lateralised modulation of $\alpha$-power $(8-14 \mathrm{~Hz})$ in the visual cortex $[18,19]$. The neurophysiological patterns underlying CVSA have been studied via the modulation of the brain responses elicited by external visual stimuli [20], as well as via the self-generated modulation of the activity in the visual cortex in a fully endogenous way (i.e., not depending on external stimuli) [21, 22]. These covert shifts of attention have also been explored in environments containing ambiguous spatial information [23]. Tonin et al. [8, 24] have tested the feasibility of detecting covert shifts of visual spatial attention in real-time by analysing $\alpha$ sub-band modulations.

\section{MATERIALS AND METHODS}

\subsection{Clip-On EEG Piece for HoloLens 2}

We have designed our own EEG clip-on holder for HoloLens 2 to host all the electrodes and electronics for the brain-sensing component of our system. The HoloLens 2 EEG clip-on had three major considerations: fit, effectiveness and material. It had to accomplish three things in particular: (1) Conform to the shape of the HoloLens 2 front and back headset parts; (2) Ensure that the EEG electrodes form a strong physical contact with the scalp; (3) The clip-on holder piece fits rather comfortably for the user. The clip-on piece for the HoloLens 2 includes three parts: the first two parts house EEG electrodes that make contact with the forehead and the back of the head, while the last part, attached at the back of the HoloLens 2, holds the electronics (Fig. 1). The first round of ideation focused on finding an optimal shape that maximizes the contact area. We explored a number of geometric patterns that can be used to house up to eight electrodes on each part, and found the staggering pattern to be the most effective. The teardrop-shaped EEG sensors would sit inside the extruded cut curvature of the clip-on.

The HoloLens 2 clip-on was designed in Solidworks and later 3D printed. The extrude command was used to create curvature for the pieces that was then extrude cut to make teardrop slots that are used to hold the EEG electrodes. Following the computer model, the CAD models were 3D printed with thermoplastic polyurethane (TPU) filament, which is flexible and abrasion resistant. We have chosen TPU material over polylactic acid (PLA) because of its flexible material properties.

\subsection{Participants}

14 adult participants ( 7 women, 18 to 38 years old, median age: 23 years) with normal or corrected-to-normal vision participated in this study. The study was approved by the ethics committee of MIT where the study took place. The participants were recruited among the campus passers-by and students, and they did not receive any compensation for their participation.

\subsection{Visuospatial Attention Protocol}

We followed the protocol from Tonin's et al., work [8], which used a modified version of Posner's spatial cueing task [25] (see Fig. 2). A white fixation cross in the center of a screen and two white circles positioned at the bottom-left and bottom-right locations were displayed continuously. After $2000 \mathrm{~ms}$ of fixation, a cue (arrow) was displayed for $100 \mathrm{~ms}$ indicating on which of the two target locations (circles) the user is supposed to focus their attention. Users were instructed to perform the task without overt eye movements. After 3000-5000 ms of sustained covert attention, a red disc appeared over one of the target locations, indicating the result of the classification (feedback) and indicating the end of the trial. During the calibration phase the feedback always appeared at the correct, i.e. cued, location. The duration of the covert attention period was randomized to avoid the user adapting to the protocol pace. During the covert attention period no external stimuli were provided to the users. They were instructed to restrain from blinking or moving their eyes as much as possible until the end of each trial.

\subsection{Experimental Design}

Each user participated in two recording sessions separated by 1 to 4 days (depending on the availability of each user to come back to the lab). On the first day users performed four calibration runs (total of 160 trials, 40 trials/run). The calibration phase lasted on average $39.8 \pm 6.2$ minutes. After a break $(10 \pm 5 \mathrm{~min})$, the users performed four real-time runs (total of 120 trials, 30 trials/run). The classifier used for the real-time runs was built with the data from the calibration phase and then updated with the first two real-time runs. On the second day of the experiment, the subjects performed four additional real-time runs (total of 120 trials, 30 trials/run) using the same classifier from the previous day. In total, each user performed 400 trials across the two recording days. Trials were randomly shuffled between the two classes.

\subsection{Data Acquisition and Preprocessing}

We used the Microsoft HoLoLens 2 headset as an AR HMD platform. We used electrodes from the g.tec Unicorn Hybrid system [26]. We 


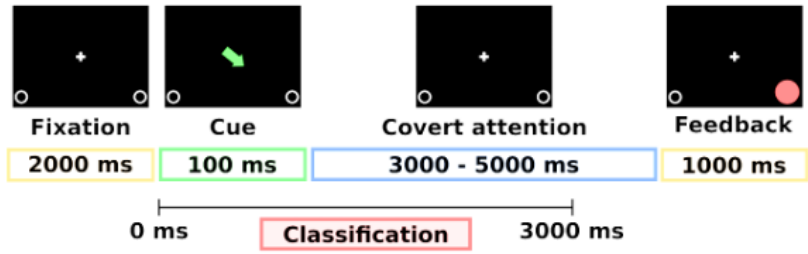

Figure 2: Experimental design paradigm.

attached the Unicorn electrodes (any electrodes can be used) to HoloLens 2 by 3D-printing a clip-on holder (Fig. 1, in blue), which contains all the electrodes and electronics pertaining to EEG. Thus, we discarded the use of any caps which are usually an integral part of g.tec Unicorn system and many other commercially available BCI systems.

We acquire EEG from 14 dry EEG electrodes. The data is sampled with $24 \mathrm{Bit}, 250 \mathrm{~Hz}$ per channel. The electrodes were placed according to the standard international 10-20 system on prefrontal and the parieto-occipital regions of the brain: FP1, FPz, FP2, AF7, $\mathrm{AF} 1, \mathrm{AF} 2, \mathrm{AF} 8$ and PO7, PO3, POz, PO4, PO8, O1, O2. Reference and ground electrodes were fixed on the mastoids of the user. After acquisition, the EEG data of a given trial was low-pass filtered by cutting-off frequency at $200 \mathrm{~Hz}$. The envelope of the signals was computed for seven frequency sub-bands in the $\alpha$ range. Subsequently, a Laplacian spatial filter was applied. Please check [8] for more details.

Unlike, [8], our experiment was conducted in the 3D mixed reality environment instead of a 2D screen. We used Unity 3D as the software to set up the scenes for covert visuospatial attention experiment setup. The HoloLens 2 has built-in eye-tracking functionality which allowed us to know what the user was looking at. It provides eye-gaze ray (gaze origin and direction) data at approximately 30FPS.

\subsection{Real-Time BCI Classification}

In order to discriminate between the two classes (the left or right locus of attention) we adopted a time-dependent classification method following [8]: the classification of every trial was based only on the data from the first $3000 \mathrm{~ms}$ after cue onset. This period was split into consecutive non-overlapping windows of $150 \mathrm{~ms}$. For each window we built a quadratic discriminant analysis classifier. Please check [24] for details. During real-time runs each classifier analyzed its corresponding window sequentially: we accumulated the posterior probabilities of each time window in a Bayesian framework in order to make the final decision about the trial [27]. The real-time accuracy of the BCI was evaluated in terms of the percentage of correctly classified trials.

\subsection{Offline Analysis: Gaze Control}

After the completion of the online sessions, the data was additionally analyzed offline in order to further investigate the performance of the system. As one of the motivations of our setup was investigating the EEG acquisition and analysis to enable real-time use cases, real-time BCI classification must make predictions on the trials as they are received. Offline clean-up stages cannot be used. But following Tonin et al.'s work [8] and rather novel hardware setup, we evaluated the possible influence of eye movements. Using eye-tracker data we: (1) quantified the incidences of overt gaze visits to the target locations and (2) investigated the possible influences of eye movements on the classification.

We defined two circular regions of interest (RoI) covering $8.8^{\circ}$ of the visual field, centered on the target locations. This number was calculated based on the maximum distance of the two circular regions. We conservatively considered that a target location received an overt glance (saccades) if the subject's gaze visited the corresponding RoI continuously for at least $50 \mathrm{~ms}$.

However, to additionally verify whether any shifts in gaze direction could influence or account for our BCI classification results, we performed classification of the trials based solely on eye movements. The HoloLens 2 has 2 IR cameras and provides real-time eye-tracking data such as GazeOrigin on $\mathrm{x} / \mathrm{y} / \mathrm{z}$-axis, GazeDirection on $\mathrm{x} / \mathrm{y} / \mathrm{z}$-axis, and information about the currently gazed-at target (HitInfo, HitPosition and HitNormal) [28]. For eye-tracking data collected from the HoloLens 2, we extracted the horizontal component $\mathrm{x}$ and vertical component $\mathrm{y}$ of the gaze location. We set the fixation cross as the origin $(0,0)$. The horizontal coordinate value gave us a metric specifying if the user was looking to the left or to the right. The vertical coordinate value gave us a metric specifying if the user was looking up or down. The trials were classified as left or right based on this metric.

Subsequently, for each user a $\varphi$ coefficient was calculated between a vector of gaze-based trial-by-trial classifications (correct or incorrect, 1 or 0 ) and a similar vector for BCI classification. The $\varphi$ coefficient is a measure of association between two variables; it can be considered as an equivalent of Pearson's correlation coefficient for binary variables. A high association between gaze-based and BCI classifications would mean that both performed well or both failed, thus suggesting that both rely on the same physiological processes-correlates of overt shifts in gaze direction. A low or no association would demonstrate that BCI classification detects cortical processes which are generally independent of incidental gaze shifts.

\section{RESULTS}

\subsection{Online (Real-time) BCI Performances}

Figure 3 shows the online performances for each user averaged over the two days of the experiment. All users performed better than a chance level of $p<0.05$. To double-check if the classification results are significantly different from what could be achieved by chance, we follow Muller-Putz et al. [29]. They propose that the reported accuracies should be compared against the upper bound of a confidence interval around the expected accuracy of a random classifier. Accuracy averages inside the confidence interval cannot be rejected from being essentially random. The same approach has been followed by $[8,24,29,30]$. The interval bounds depend on the amount of trials in the experimental design. In the accuracy plots we present, a horizontal line denotes the upper boundary for the confidence interval with a threshold $p<0.05$ as obtained from Muller-Putz et al. [29]. The overall accuracy across subjects and runs was $70.0 \pm 5.5 \%$ (mean \pm standard error of the mean (SEM)). 


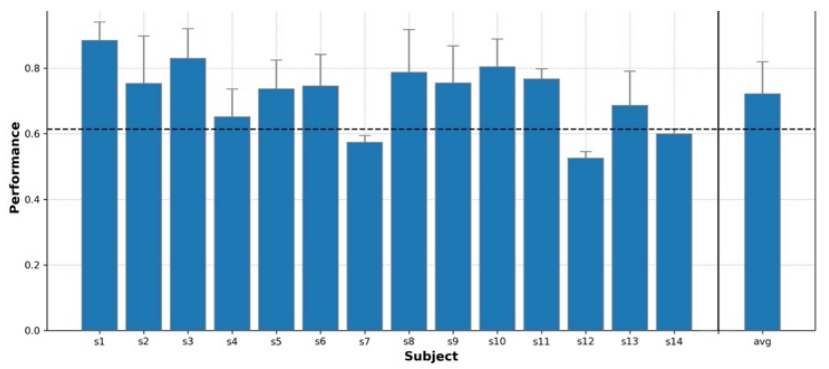

Figure 3: Online performance of each user averaged over the two days of the experiment. Error bars denote standard deviation of the mean. The broken line indicates the chance level of $p<0.05$.

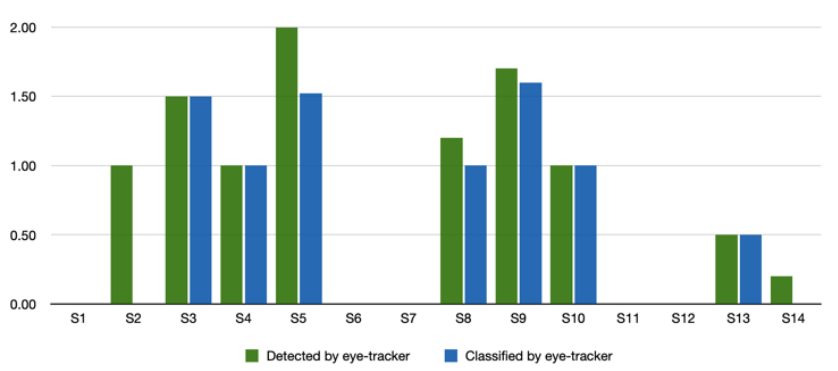

Figure 4: Overt eye movements: percentage of trials involving overt gaze shifts: (1) towards either one of the target locations (green bars); (2) towards just the cue target location (blue bars).

\subsection{Gaze Shifts}

In Fig. 4 we present the percentage of trials that involved overt gaze direction shifts towards either one of the target locations (green bars). This percentage is very low for all subjects: $0.73 \%$ on average, maximum $2 \%$; it is $0 \%$ for subjects $\mathrm{s} 1, \mathrm{~s} 6, \mathrm{~s} 7, \mathrm{~s} 11, \mathrm{~s} 12$ and below $1 \%$ for s 13 and s 14 . As expected, most of the eye movements were directed towards the cued target location (blue bars in the figure). Given the small number of contaminated trials it can be assumed that gaze shifts had a negligible impact in the classification accuracy. Additionally we investigated the association between classification based on the prevailing gaze direction in a given trial and EEGbased BCI classification. We found a low correlation for all subjects (Fig. 5), further confirming that BCI classification detects cortical processes which are independent of any occurring gaze shifts.

\section{DISCUSSION}

In this study we demonstrated the online, real-time setup of using an AR EEG-BCI integrated prototype based on CVSA not relying on any external stimulation. Online classification accuracy of 70.0 $\pm 5.5 \%$ (averaged across subjects and recording sessions) proves CVSA to be in line with current percentages of control signal for $\mathrm{BCI}$ applications as well as the similar state of the art research projects $[8,22-24]$, all reporting between $69 \%$ and $74 \%$.

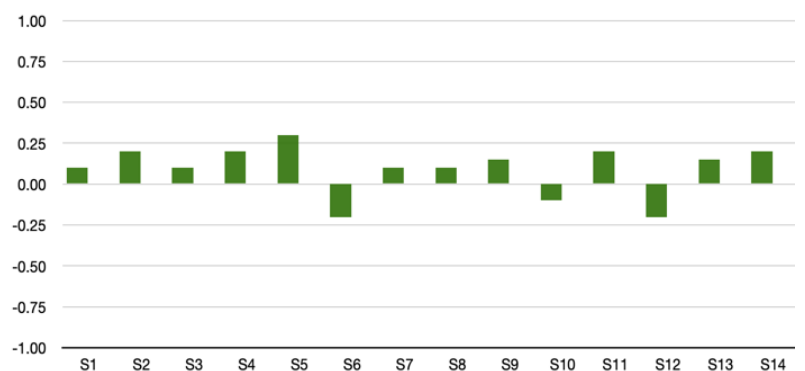

Figure 5: Overt eye movements: correlations ( $\varphi$ coefficients) between the trial-by-trial accuracy of classification based on the horizontal component of eye saccades and BCI; none significant at $\boldsymbol{p}<\mathbf{0 . 0 5}$.

The proof-of-concept presented in this paper opens up interesting possible applications of AR EEG-BCIs based on CVSA. Inherent gaze independence of CVSA makes it a promising alternative for completely locked-in patients, who do not display any overt eye movements. Its intuitiveness-natural attraction toward regions or objects of interest in the visual field-makes it a promising candidate for BCI-driven navigation devices (e.g., wheelchairs or robots), as well as yes-no communication. Absence of stimulation stimuli like ERPs/SSVEPs may prove it more suitable for use over longer periods of time, as it allows a more engaging and direct operation, and it is more adapted towards the out-of-lab interactions. However, the results of this study should be considered as preliminary and the effect of extended use of such integrated systems based on locus of attention should be explored further. Another interesting approach lies in coupling CVSA with other BCI control signals in a hybrid framework-particularly with another non-time-locked paradigms like motor imagery. Advantages include the fact that both rely on independent EEG correlates, while offering isolated scalp distributions. Such hybrid system could be implemented in order to increase the number of available commands or to decrease the uncertainty of the existing ones.

Finally, a prospect of enhancing AR interaction using CVSA also appears to be intriguing, in the applications where the user might need additional modality to access some hidden properties of the system without evoking those in an explicit manner.

\section{REFERENCES}

[1] Liesjet van Dokkum, Tomas E. Ward and Isabelle Laffont. Brain computer interfaces for neurorehabilitation - its current status as a rehabilitation strategy post-stroke. Annals of Physical and Rehabilitation Medicine, Volume 58, Issue 1, 2015, Pages 3-8, ISSN 1877-0657.

[2] Karl LaFleur, Kaitlin Cassady, Alexander Doud, Kaleb Shades, Eitan Rogin, and Bin He. 2013. Quadcopter control in three- dimensional space using a noninvasive motor imagery-based brain-computer interface. J. Neural Eng. 10, 4 (2013), 046003. https://doi.org/10.1088/1741-2560/10/4/046003

[3] Daniel Afergan, Evan M. Peck, Erin T. Solovey, Andrew Jenkins, Samuel W. Hincks, Eli T. Brown, Remco Chang, and Robert J.K. Jacob. 2014. Dynamic difficulty using brain metrics of workload. In Proceedings of the SIGCHI Conference on Human Factors in Computing Systems (CHI '14). Association for Computing Machinery, New York, NY, USA, 3797-3806.

[4] Nataliya Kosmyna, Franck Tarpin-Bernard, and Bertrand Rivet. 2015. Towards brain computer interfaces for recreational activities: Piloting a drone. In Proceedings of INTERACT 2015. Bamberg, Germany.

[5] Nataliya Kosmyna and Franck Tarpin-Bernard. 2013. Evaluation and comparison of a multimodal combination of BCI paradigms and eye tracking with affordable consumer-grade hardware in a gaming context. IEEE Trans. Comput. Intell. AI 
Games 5, 2 (2013), 150-154.

[6] Judith Amores, Robert Richer, Nan Zhao, Pattie Maes, and Bjoern M Eskofier 2018. Promoting relaxation using virtual reality, olfactory interfaces and wearable EEG. In 2018 IEEE 15th International Conference on Wearable and Implantable Body Sensor Networks (BSN). IEEE, 98- 101.

[7] Nathan Semertzidis, Michaela Scary, Josh Andres, Brahmi Dwivedi, Yutika Chandrashekhar Kulwe, Fabio Zambetta, and Florian Floyd Mueller. 2020. NeoNoumena: Augmenting Emotion Communication. In Proceedings of the 2020 CHI Conference on Human Factors in Computing Systems (CHI '20). ACM, New York, NY, USA, 1-13.

[8] Luca Tonin, Robert Leeb, Aleksander Sobolewski and Josed́el R. Millań. 2013. An online EEG BCI based on covert visuospatial attention in absence of exogenous stimulation. J. Neural Eng. 10 056007, 2013.

[9] https://www.microsoft.com/en-us/hololens/hardware

[10] F. Putze, D. Wei $\beta$, L. Vortmann and T. Schultz, "Augmented Reality Interface for Smart Home Control using SSVEP-BCI and Eye Gaze," 2019 IEEE International Conference on Systems, Man and Cybernetics (SMC), Bari, Italy, 2019, pp. 2812 2817.

[11] H. Si-Mohammed et al., "Towards BCI-Based Interfaces for Augmented Reality: Feasibility, Design and Evaluation," in IEEE Transactions on Visualization and Computer Graphics, vol. 26, no. 3, pp. 1608-1621, 1 March 2020.

[12] Guillermo Bernal, Tao Yang, Abhinandan Jain, and Pattie Maes. 2018. PhysioHMD a conformable, modular toolkit for collecting physiological data from headmounted displays. In Proceedings of the 2018 ACM International Symposium on Wearable Computers(ISWC '18). Association for Computing Machinery, New York, NY, USA, 160-167.

[13] K. Takano, N. Hata, and K. Kansaku, "Towards intelligent environments: An augmented reality brain-machine interface operated with a see-through headmount display," Frontiers in Neuroscience, vol. 5, p. 60, 2011. [Online]. Available: http://journal.frontiersin.org/article/10.3389/fnins.2011.00060

[14] Lisa-Marie Vortmann and Felix Putze. 2020. Attention-Aware Brain Computer Interface to Avoid Distractions in Augmented Reality. In Extended Abstracts of the 2020 CHI Conference on Human Factors in Computing Systems (CHI EA '20). ACM, New York, NY, USA, 1-8.

[15] Meng Wang, Renjie Li, Ruofan Zhang, Guangye Li, and Dingguo Zhang. 2018. A Wearable SSVEP-Based BCI System for Quadcopter Control Using Head-Mounted Device. IEEE Access (2018).

[16] Escolano C, Antelis JM, Minguez J. A telepresence mobile robot controlled with a noninvasive brain- computer interface. IEEE SMC. 42, 3 (2012), 793-804.

[17] L. Angrisani, P. Arpaia, N. Moccaldi and A. Esposito, "Wearable Augmented Reality and Brain Computer Interface to Improve Human-Robot Interactions in Smart
Industry: A Feasibility Study for SSVEP Signals," 2018 IEEE 4th International Forum on Research and Technology for Society and Industry (RTSI), Palermo, 2018, pp. 1-5.

[18] Sauseng, P. et al. A shift of visual spatial attention is selectively associated with human EEG alpha activity. Eur. J. Neurosci. 22, 2917-2926 (2005).

[19] Rihs, T. A., Michel, C. M. \& Thut, G. A bias for posterior a-band power suppression versus enhancement during shifting versus maintenance of spatial attention. Neuroimage 44, 190-199 (2009).

[20] Schmidt, N., Blankertz, B. \& Treder, M. S. $\alpha$-modulation induced by covert attention shifts as a new input modality for EEG-based BCIs. In Systems Man and Cybernetics (SMC), 2010 IEEE International Conference on, 481-487 (IEEE, 2010).

[21] Treder, M. S., Schmidt, N. M. \& Blankertz, B. Gaze-independent brain-computer interfaces based on covert attention and feature attention. J. Neural Eng. 8, 066003 (2011).

[22] Treder, M. S., Bahramisharif, A., Schmidt, N. M., Van Gerven, M. A. \& Blankertz, B. Brain-computer interfacing using modulations of alpha activity induced by covert shifts of attention. J. Neuroeng. Rehabil. 8, 24 (2011).

[23] Trachel, R. E., Clerc, M. \& Brochier, T. G. Decoding covert shifts of attention induced by ambiguous visuospatial cues. Front. Hum. Neurosci. 9, 358 (2015).

[24] Tonin, L., Leeb, R. \& Millań, J. D. R. Time-dependent approach for single trial classification of covert visuospatial attention. J. Neural Eng. 9, 045011 (2012). Michael I. Posner. 1980. Orienting of attention, Quarterly Journal of Experimental Psychology, 32:1, 3-25.

[25] https://www.unicorn-bi.com

[26] Beck J M, Ma W J, Kiani R, Hanks T, Churchland A K, Roitman J, Shadlen M N, Latham P E and Pouget A 2008 Probabilistic population codes for Bayesian decision makingNeuron601142-52

[27] https://microsoft.github.io/MixedRealityToolkit-Unity/Documentation/ EyeTracking/EyeTracking_EyeGazeProvider.html

[28] Muller-Putz, G., Scherer, R., Brunner, C., Leeb, R. \& Pfurtscheller, G. Better than Random? A closer look on BCI results. International Journal of Bioelektromagnetism. 10, 52-55 (2008).

[29] Kosmyna, N., Lindgren, J.T. \& Lécuyer, A. Attending to Visual Stimuli versus Performing Visual Imagery as a Control Strategy for EEG-based Brain-Computer Interfaces. Sci Rep8, 13222 (2018). https://doi.org/10.1038/s41598-018-31472-9

[30] Nataliya Kosmyna, Qiuxuan Wu, Chi-Yun Hu, Yujie Wang, Cassandra Scheirer, Pattie Maes. Assessing Internal and External Attention in AR using Brain Computer Interfaces: A Pilot Study. In Proceedings of 2021 International Conference on Wearable and Implantable Body Sensor Networks (IEEE BSN '21). Institute of Electrical and Electronics Engineers, USA. 